\title{
An Advanced 3D Multi-Body System Model for the Human Lumbar Spine
}

\author{
Sousa, S. ${ }^{1}$ and Claro, J. C. P. ${ }^{2}$ \\ ${ }^{1}$ University of Minho,Portugal,e-mail: sofia_sts@hotmail.com \\ ${ }^{2}$ University of Minho, Portugal, e-mail: jcpclaro@dem.uminho.pt
}

\begin{abstract}
A novel 3D multi-body system model of the human lumbar spine is presented, allowing the dynamic study of the all set but also to access mechanical demands, characteristics and performance under work of the individual intervertebral discs. An advanced FEM analysis was used for the most precise characterization of the disc 6DOF mechanical behavior, in order to build up a tool capable of predicting and assist in the design of disc recovery strategies - namely in the development of replacement materials for the degenerated disc nucleus - as well as in the analysis of variations in the mechanical properties (disorders) at disc level or kinematic structure (e.g. interbody fusion, pedicle fixation, etc.), and its influence in the overall spine dynamics and at motion segments individual level. Preliminary results of the model, at different levels of its development, are presented.
\end{abstract}

Key words: computer simulation, multibody systems, biodynamics, spine column

\section{Introduction}

Low back pain affects about $80 \%$ of world's population, at some point of their life, been lumbar disc diseases and, among them, the degeneration of the disc's nucleus pulposus (NP) and consequent loss of internal pressure, one of the main causes [1]. Current treatments, if not just palliative, present multiple and severe side effects, which lead the research to look for reliable prosthetic solutions for partial or complete substitution of the nucleus, not only as a viable alternative but also, and preferably, involving minimum invasive and lower traumatic techniques and shorter recovery time and ambulatory periods for the patients.

It's with this background that a 'in silica' study of the working conditions of the intervertebral disc (IVD) - as part of a broader research project (NPMimetic.com) - was developed. In a first stage as a way to characterize and quantify the mechanical behavior of the IVD bio-system, through a Finite Elements Method (FEM) and, in a second phase, to analyze the IVD performance 'de 
per si', as well as eventual influences in the global dynamics of the spinal system, using a Multi-Body System (MBS) analysis.

The option for the MBS model was justified by its considerably low computational demand, when compared to FEM for instance, but also for its versatility in the definition of complex dynamic loading conditions and the ease of introducing eventual variations on the characteristics on specific components, allowing stable and reliable solutions in the dynamic analysis of a complex system, involving the IVD but also the main ligaments acting on mechanical stabilization of the spine.

The model is intended to provide data about IVD's 3D loading state for normal daily life activities, as well as in the full physiological range, allowing the analysis, quantification and systematization of its 'real' performance conditions, been in natural physiological conditions or with a partial or total replacement of the NP by a prosthetic material. Subsequently, these data will be used as a feedback to the FEM model, allowing its detailed study at nucleus/annulus level and the verification and possible re-engineering of the prosthetic material.

Besides the prediction of the effect of alterations in the mechanical characteristics of the NP due to an implant, this MBS tool is also able to predict the effects of other local alterations - such as those induced by a motion segment fusion, by chirurgical intervention, or due to degeneration/ageing phenomena, for instance - allowing the quantification of the (clinical well known) change on the loading pattern, induced in the neighborhood levels of the spine.

Furthermore, it can also be useful in the design of mechanical devices for spine stabilization, namely in the most recent and yet in development partially mobile, mechanical driven, linkage systems.

\section{Implementation}

The MBS model, implemented in a commercial software (WM-VisualNastran), has six rigid bodies representing the five lumbar vertebrae and the sacrum, interconnected through fifty non-linear Maxwell elements (spring/damper parallel sets) emulating the twenty main ligaments [2] and five IVDs, besides the five bilateral posterior spinous processes (for the moment defined as simple spatial constrains, with no specific surface contact law) in a complete lumbar set as shown in Fig.1.

For the mechanical characteristics of the IVD, data generated by an in-house advanced osmo-porous-hiper-viscoelastic FEM model, specifically developed within the "NP Mimetic" project [3, 4], were used in the prediction of the mechanical behavior in translation (Tx, Ty and $\mathrm{Tz}$ ) and in rotation (Rx, Ry and Rz) along the three coordinated axis of the medial plane of the IVD. For the ligaments, published available data were used, giving a special attention to those that are more relevant for the stability and performance of the spine - namely the longitudinal anterior (ALL) and posterior (PLL), flavum (FL), interspinous (ISL) and supraspnious (SSL) ligaments - after a careful curation and selection [5, 6, 7]. Currently, Chazal results are implemented. 

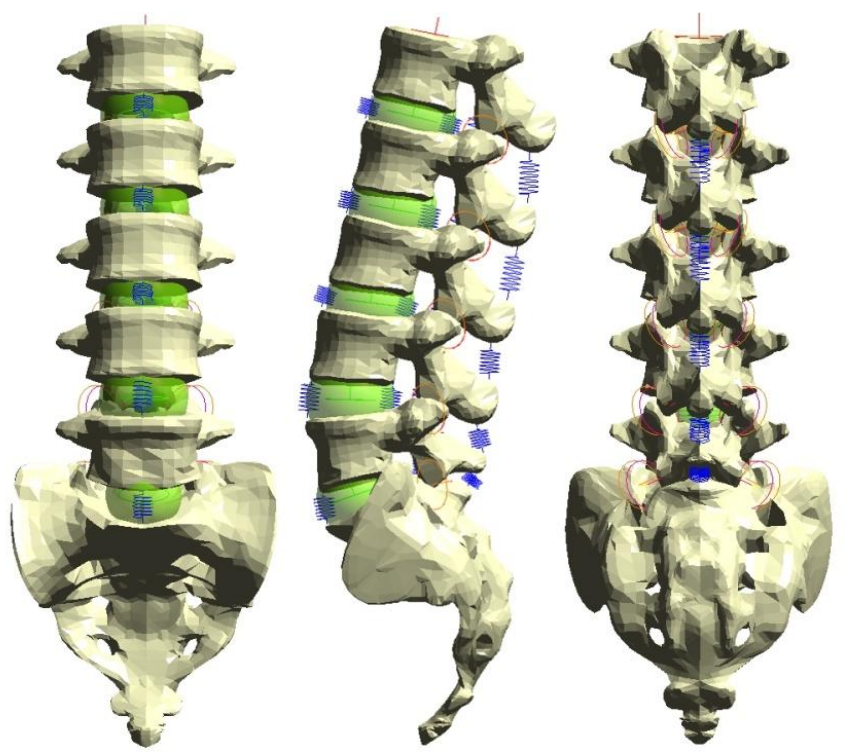

Fig.1 - Multi-body model of the human lumbar spine

Fig.2 displays a left antero-posterior perspective detail of the kinematic model scheme, where the central cube represents the IVD, with its six DOF, the helical lines stand for the ligaments and the processes defined by direct contact between adjacent bodies.

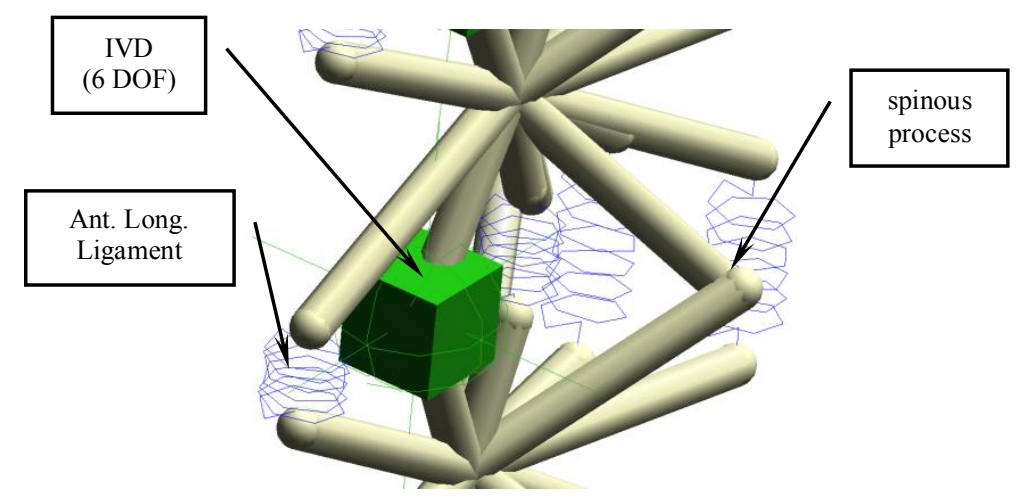

Fig.2 - Detail of the MBS joints on the motion segment

Subsequently, a throughout sensitivity study of the motion segment geometric parameters (variation of average thickness, sagittal angle between end-plates and transverse section of IVD and annulus/nucleus ratio) on IVD's behavior was car- 
ried out, and results parameterized and introduced in the force/torque versus elongation/rotation equations of the Maxwell elements, according to their different levels on the spine.

\section{Results}

Preliminary results are presented, showing the potentialities of the model in the reproduction and quantification of different circumstances, in three different phases:

a) validation of the answer of each one of the components 'de per si' - as illustrated in Figs. 3 and 4;

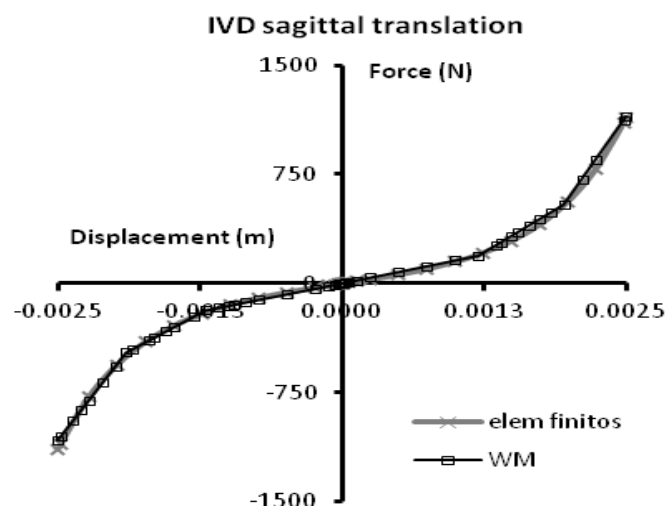

Fig.3 - Validation of the model' IVD

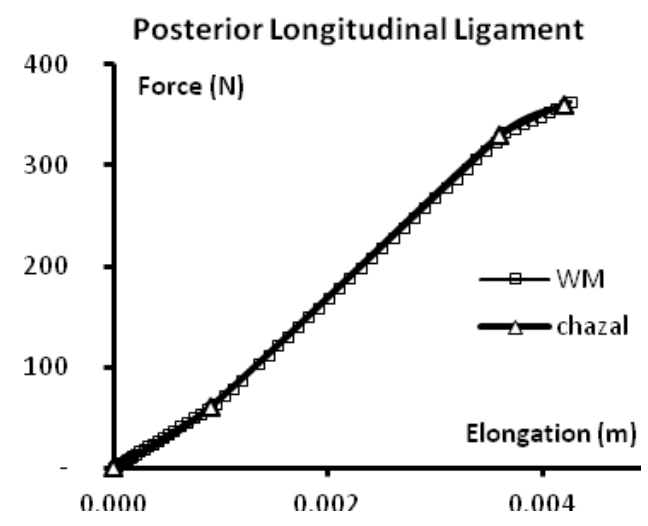

Fig.4 - Validation of model's ligaments 
b) analysis of the correlation with others experimental works [8] - Fig. 6 - and with the answer to daily-life, usual but complex composition of load cases and applied ratios, using external published data (e.g. Rolhman [9] and Orthoload [10])

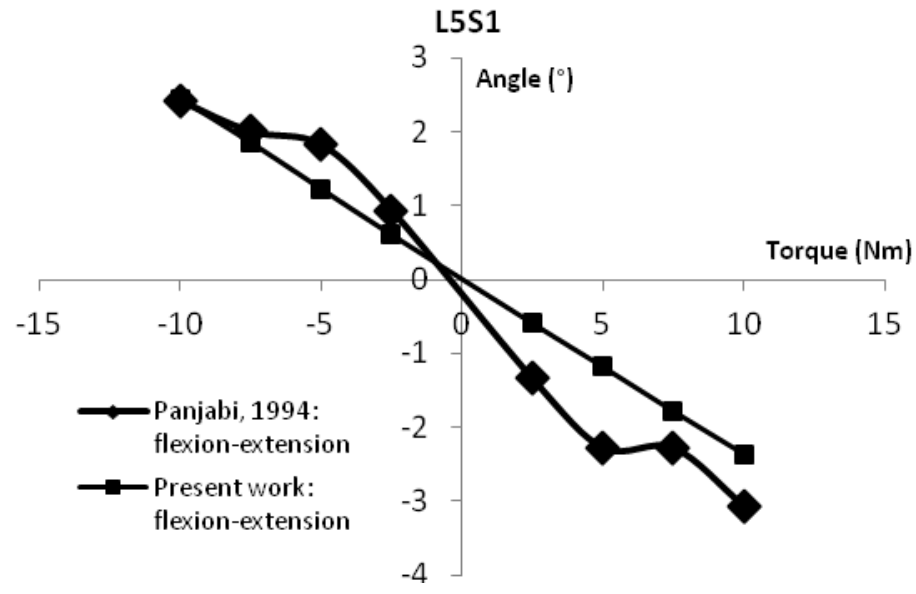

Fig.6 - Comparison with experimental data

c) study on the influence of critical parameters (namely of the functional characteristics of the IVD) and the virtualization of the 'fusion' of one motion segment, in the static and dynamic response of the other levels and of the all lumbar section - Figs. 7 and 8.

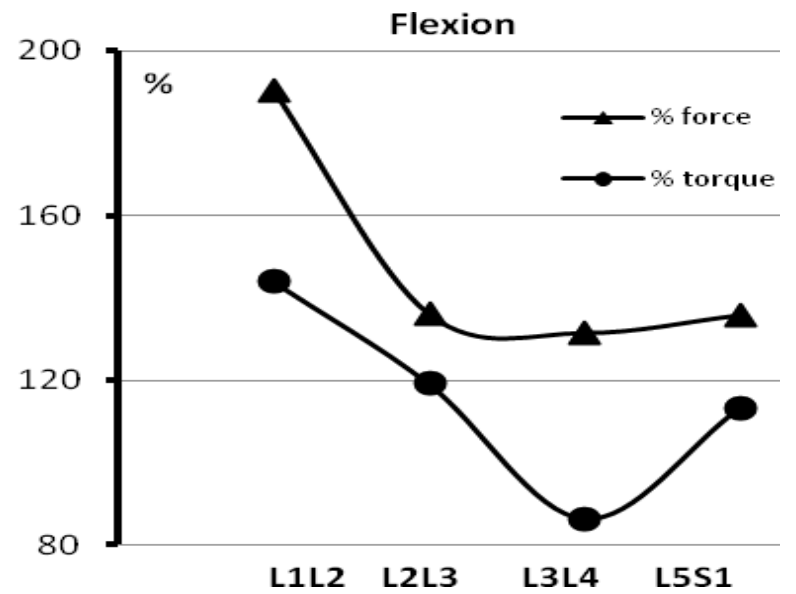

Fig.7 - Flexion: percentage increase in static load, for a L4-L5 interbody fusion 


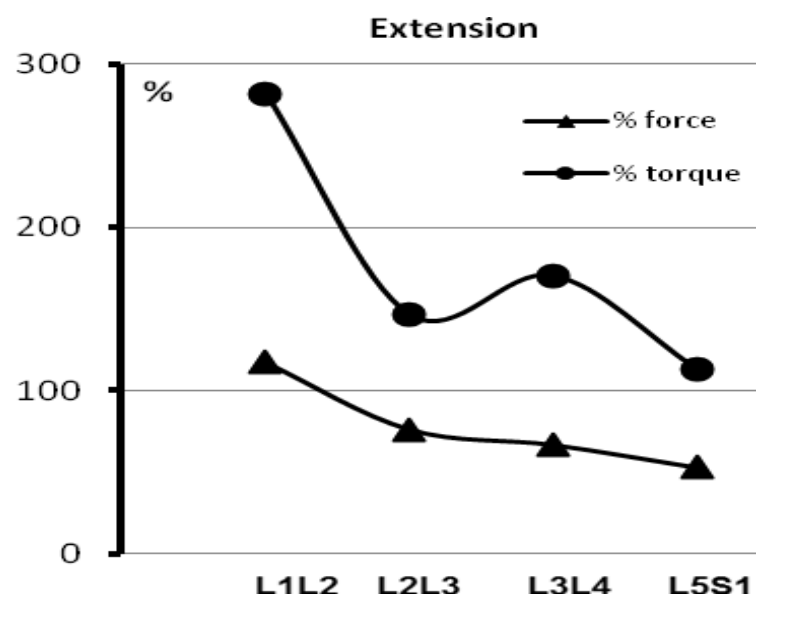

Fig.8 - Extension: percentage increase in static load, for a L4-L5 interbody fusion

\section{Conclusions}

The model is in a phase of tuning-up, to suit the needs of broader project's objectives - implying a post-processing of output data, via a FEM analysis, aiming the full mechanical design of the advanced prosthetic materials under developed for NP regeneration.

However it is presently fully validated, already shown to be a feasible and reliable, 'light' computer demanding tool, suitable for the study and analysis of several complex problems, involving various clinical problematic of the lumbar spine, and the corresponding prediction and design of mechanical solutions.

Acknowledgments This work was performed within the European Project "NP Mimetic - Biomimetic Nano-Fibre Based Nucleus Pulposus Regeneration for the Treatment of Degenerative Disc Disease", funded by the European Commission under FP7 (grant NMP-2009-SMALL-3-CP-FP 246351) 


\section{References}

1 Kraemer, J. "Intervertebral Disk Diseases: Causes, Diagnosis, Treatment and Prophylaxis, Thieme, Stuttgart, 2008

2 Behrsin, J; Briggs, C. "Ligaments of the lumbar spine: a review", Surgical and Radiologic Anatomy, 10, pp. 211-219, 1988

3 Castro, A et al (2013), "The role of poroelasticity on the biomechanics of the intervertebral disc: a finite element study". 5th. National Congress on Biomechanics, Espinho, Portugal

4 Castro, A. et al (2012), "A poroelastic approach for an open source finite element model of the intervertebral disc". Proceedings of CMBBE2012 Symposium

5 Chazal, J et al (1985), "Biomechanical properties of spinal ligaments and a histological study of the supraspinal ligament in traction". J. Biomech., vol. 18, no. 3, pp. 167-176

6 Neumann, P et al (1992) "Mechanical properties of the human lumbar anterior longitudinal ligament," J. Biomech., vol. 25, no. 10, pp. 1185-1194

7 Pintar, F A et al (1992), "Biomechanical properties of human lumbar spine ligaments". Journal of Biomechanics, 25, pp. 1351-1356

8 Panjabi, M et al. (1994) "Mechanical Behavior of the Human Lumbar and Lumbosacral Spine as Shown by Three-Dimensional Load-Displacement Curves". New Haven: The Journal of Bone and Joint Surgery, vol. 76-A (3), pp.413-423

9 Rohlmann, A et al (2007), "An instrumented implant for vertebral body replacement that measures loads in the anterior spinal column". Medical Engineering and Physics, 580-585

10 Bergmann, G. (ed.), Charite - Universitaetsmedizin Berlin (2008) "OrthoLoad". Retrieved November 06, 2012, from <http://www.orthoload.com> 